\title{
An Enhancement of Data Hiding Imperceptibility using Slantlet
} Transform (SLT)

\author{
Daurat Sinaga*1, Eko Hari Rachmawanto ${ }^{2}$, Christy Atika Sari ${ }^{3}$, De Rosal Ignatius Moses \\ Setiadi ${ }^{4}$, Noor Ageng Setiyanto ${ }^{5}$ \\ 1,2,3,4,5 Universitas Dian Nuswantoro/Departement of Informatics Engineering \\ parendeborngin@gmail.com ${ }^{* 1}$, eko.hari@dsn.dinus.ac.id ${ }^{2}$, atika.sari@dsn.dinus.ac.id ${ }^{3}$, \\ moses@dsn.dinus.ac.id ${ }^{4}$, nasetiyanto@gmail.com ${ }^{5}$
}

\begin{abstract}
This study proposes a hybrid technique in securing image data that will be applied in telemedicine in future. Based on the web-based ENT diagnosis system using Virtual Hospital Server (VHS), patients are able to submit their physiological signals and multimedia data through the internet. In telemedicine system, image data need more secure to protect data patients in web. Cryptography and steganography are techniques that can be used to secure image data implementation. In this study, steganography method has been applied using hybrid between Discrete Cosine Transform (DCT) and Slantlet Transform (SLT) technique. DCT is calculated on blocks of independent pixels, a coding error causes discontinuity between blocks resulting in annoying blocking artifact. While SLT applies on entire image and offers better energy compaction compare to DCT without any blocking artifact. Furthermore, SLT splits component into numerous frequency bands called sub bands or octave bands. It is known that SLT is a better than DWT based scheme and better time localization. Weakness of DCT is eliminated by SLT that employ an improved version of the usual Discrete Wavelet Transform (DWT). Some comparison of technique is included in this study to show the capability of the hybrid SLT and DCT. Experimental results show that optimum imperceptibility is achieved.
\end{abstract}

Keywords: Slantlet Transform, Discrete Wavelet Transform, Discrete Cosine Transform, Image

\section{Introduction}

Telemedicine is one of the applications which use Internet to communicate and share data. The telemedicine was developed based on multidisciplinary integration of information technology, network technology, medical instrumentations and clinics medicines. The focus of this study is related to there is few applications published. According to [1] described Ear-Nose-Throat (henceforth, ENT) medical image in diagnosis system to find the sender of data. Through this system, patient can be sent using virtual hospital server that take an advantage of the internet.

For the example, the patients may ask for a diagnosis at a distance location instead of going to hospital directly. Using Virtual Hospital Server (VHS), physician may process the online diagnosis in the Internet. More attackers could be eliminated by securing methods. Methods and techniques will be secured the data. Both cryptography and steganography are able to secure the data directly or indirectly.

Cryptography uses for encrypt and decrypt data so that data cannot be opened. Steganography is the art of hiding the existence data in another medium transmission to achieve secret communication. We focus in steganography to secure the data to hide into another data.

In steganography, secret message is the data that sender wishes to remain confidential and can be text, images, audio, video or any other data that can be represented by a stream of bits. The cover or host which the message is embedded and serves to hide the message called "Stego-Image" [2].

There are three characteristics to design Steganography: (a) Invisibility [2], where human eyes cannot distinguish between original and stego-image. Invisibility is also known as imperceptibility. (b) Capacity [2], where it manages to embed more data, providing that the manage remains its quality. (c) Time Localization, where the use of Slantlet (henceforth, SLT) perform of the stego-image more effectively. In addition, a good time localization property make a good representation of image [3].

Sinaga, D., Rachmawanto, E., Sari, C., Setiadi, D., \& Setiyanto, N. (2018). An Enhancement of Data Hiding Imperceptibility using Slantlet Transform (SLT). Kinetik: Game Technology, Information System, Computer Network, Computing, Electronics, and Control, 4(1). doi:http://dx.doi.org/10.22219/kinetik.v4i1.702

Receive September 14, 2018; Revise November 08, 2018; Accepted November 9, 2018 
Additionally, embedded secret data is performed using frequency domain approach - SLT and DCT. Secret data is hidden in one of the high frequency sub-band of SLT by tracing skin pixels in that sub-band or octave-band.

\section{Research Method}

\subsection{Slantlet Transform}

SLT is an equivalent from DWT but provides better time-localization because the shorter support of component filter [4]. DWT usually implemented in form of an iterated bank with tree structure, but SLT draws its inspiration from an equivalent form of parallel structure with parallel branches [5]. SLT has been provided a filterbank which less frequency selective than DWT, although SLT provides better time localization compared to DWT [6].

SLT has an equivalent structure, which the scale of dilation factor is in 2 for each filterbank. SLT with two scale filterbank are given in Figure 1, while SLT with three filterbank can be displayed in Figure 2.

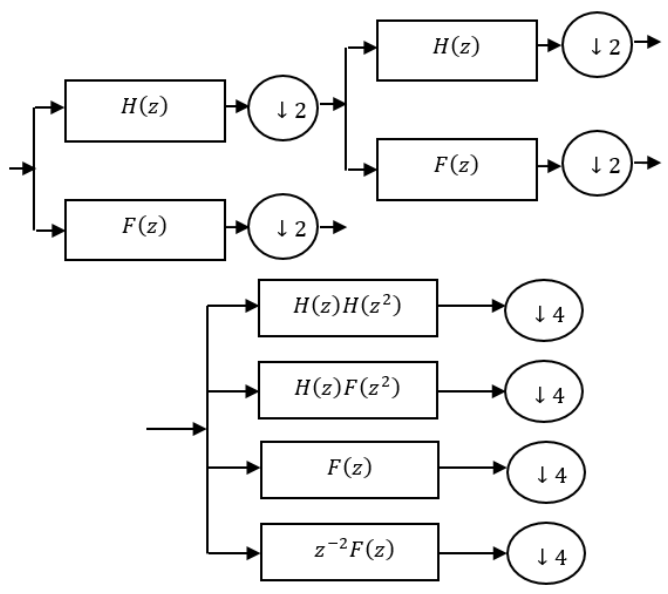

Figure 1. Two-scale Filterbank and an Equivalent Structure [3]

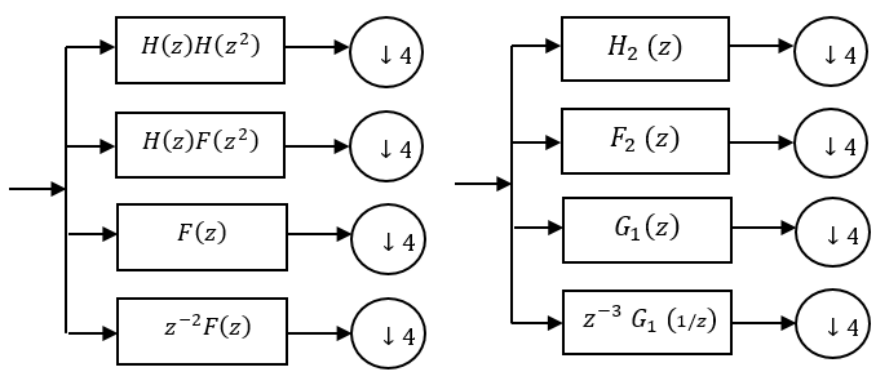

Figure 2. Comparison of Two-Scale Iterated $\boldsymbol{D}_{2}$ Filterbank (left) and Two-Scale Filterbank (Right) [3]

The outputs are down sample by a factor of 4 which are the transform coefficients then threshold using suitable parameter. The Inverse Slantlet Transform (ISLT) is performing to reconstruct the original data on this threshold [1].

Figure 2 can be described in formula that contains the filterbank fragmentations. The SLT mathematic model will be shown through the filter scale. SLT display $2^{l}$ of downsampling which is presents in channel. Assumed for $g_{i}(n), f_{i t} i(n)$ and $h_{i}(n)$ are the SLT filters that has an exact support of $2^{i+1}$. SLT has low pass filter which namely $h_{i}(n)$ and the other one is $f_{i}(n)$. Every filter conducted by $2^{l}$ downsampling. The piecewise linear of SLT will be represented as follow in Equation 1 to 3.

$$
g_{i}(n)= \begin{cases}a_{0,0}+a_{0,1} n, & \text { for } n=0, \ldots, 2^{i-1} \\ a_{0,0}+a_{0,1} n, & \text { for } n=2^{i}, \ldots, 2^{i+1}-1\end{cases}
$$

KINETIK Vol. 4, No. 1, February 2019: 87-98 


$$
\begin{aligned}
& h_{i}(n)= \begin{cases}b_{0,0}+b_{0,1} n, & \text { for } n=0, \ldots, 2^{i-1} \\
b_{0,0}+b_{0,1} n, & \text { for } n=2^{i}, \ldots, 2^{i+1}-1\end{cases} \\
& f_{i}(n)= \begin{cases}c_{0,0}+c_{0,1} n, & \text { for } n=0, \ldots, 2^{i-1} \\
c_{0,0}+c_{0,1} n, & \text { for } n=2^{i}, \ldots, 2^{i+1}-1\end{cases}
\end{aligned}
$$

Which $a s, b s$, and $c s$ used as the notation to produce the scale. The satisfaction of orthogonality and two vanishing moments will be done to present as follow in Equation 4 to 15 .

Each of $g_{i}(n), f_{i}(n)$ and $h_{i}(n)$ is of unit norm

$$
\begin{aligned}
& \sum_{n=0}^{2^{i+1}-1} g_{i}^{2}(n)=1 \\
& \sum_{n=0}^{2^{i+1}-1} f_{i}^{2}(n)=1 \\
& \sum_{n=0}^{2^{i+1}-1} h_{i}^{2}(n)=1
\end{aligned}
$$

$g_{i}(n)$ is orthogonal to its shifted time reverse

$$
\sum_{n=0}^{2^{i+1}-1} g_{i}(n) g_{i}\left(2^{i+1}-1-n\right)=0
$$

Each of $g_{i}(n)$ and $f_{i}(n)$ annihilates linear discrete time polynomials

$$
\begin{gathered}
\sum_{n=0}^{2^{i+1}-1} g_{i}(n)=0 \\
\sum_{n=0}^{2^{i+1}-1} n g_{i}(n)=0 \\
\sum_{n=0}^{2^{i+1}-1} f_{i}(n)=0 \\
\sum_{n=0}^{2^{i+1}-1} n f_{i}(n)=0
\end{gathered}
$$

$f_{i}(n)$ and $h_{i}(n)$ are orthogonal to their shifted versions

$$
\begin{gathered}
\sum_{n=0}^{2^{i+1}-1} f_{i}(n) f_{i}\left(n+2^{i}\right)=0 \\
\sum_{n=0}^{2^{i+1}-1} h_{i}(n) h_{i}\left(n+2^{i}\right)=0
\end{gathered}
$$




$$
\begin{gathered}
\sum_{n=0}^{2^{i+1}-1} h_{i}(n) f_{i}(n)=0 \\
\sum_{n=0}^{2^{i+1}-1} h_{i}(n) f_{i}\left(n+2^{i}\right)=0
\end{gathered}
$$

The SLT filterbank gives a reduction of $\left(2^{i}-2\right)$ sanples or supports for scale $i$, compared to iterated $D_{2}$ DWT filterbank, and the reduction in support approaches one third as $i$ increases. Sushil Kumar and S.K. Muttoo [4] [7] [8] [9] describes the advantages of Slantlet Transform (SLT) are better than DWT, Haar Wavelet Transform and Contourlet Tranform in image quality, best payload, get better result for extracting and embedding the original image, increase embedding capacity, and get imperceptibility.

\section{Results and Discussion}

The original image is in $512 \times 512$ pixel grayscale medical images. These, image will be shown as follow in Figure 3.
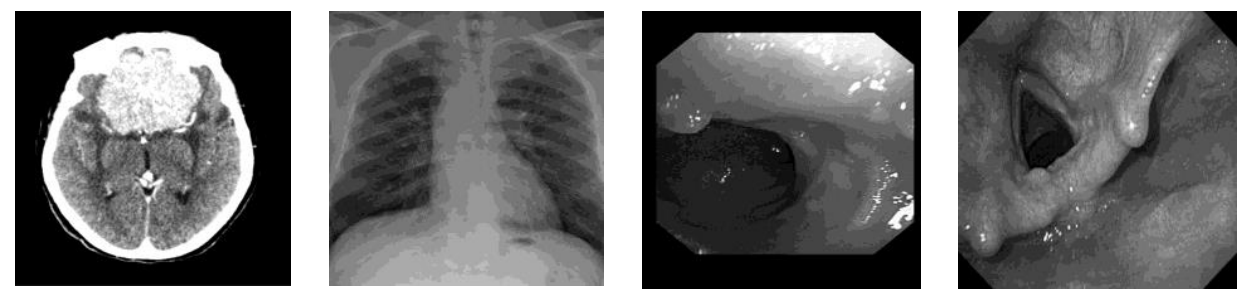

Figure 3. Tested Images Database process:

Here, we proposed a scheme to improve imperceptibility both in embedding and exctracting

\subsection{Embedding Scheme}

1. Step 1: Perform SLT on the original image to decompose it into four non-overlapping multiresolution coefficient sets: $\mathrm{LL}, \mathrm{HL}, \mathrm{LH}$ and $\mathrm{HH}$.

2. Step 2: Perform DCT to each block in step 1, and then obtain DCT domain frequency bands in the chosen sub-band (LL) coefficient. These coefficients sets are chosen to inquire imperceptibility of algorithms equally.

3. Step 3: Using Inverse DCT (IDCT) on the selected block of low-band coefficients have been modified to include the stego bit as described in the previous step.

4. Step 4: The SLT transformed image converted to stego host image using Inverse SLT (ISLT).

\subsection{Extracting Scheme}

1. Step 1: Apply SLT to decompose the steganography image into four non-overlapping multiresolution sub-bands: $\mathrm{LL}, \mathrm{HL}, \mathrm{LH}$ and $\mathrm{HH}$.

2. Step 2: Apply DCT to each block in the chosen sub-band (LL), and extract the mid-band coefficients of each DCT transformed block.

3. Step 3: Reconstruct the stego using the extracted stego bits, and compute the similarity between the original and extracted message.

\subsection{Performance Measurement}

The one of evaluation criteria from this experiment is come from the value PSNR. The high value of PSNR represented a good imperceptibility.

Figure 4 shows the result of PSNR value in Brain_Gray.jpg using five different algorithms. DCT algorithm gives 10.6298 PSNR value, DWT algorithm gives 22.3170 PSNR value, while SLT algorithm gives value of $24.9780 \mathrm{~dB}$ which is slightly same with the result given by DWT-DCT algorithm $(24.8973 \mathrm{~dB})$. The purpose algorithm of this research which is SLT-SCT gives the best value (30.7012) compare with the other algorithm from the previous research.

KINETIK Vol. 4, No. 1, February 2019: 87-98 
Whereas the result of PSNR value in Endoscopic_Gray.jpg is shown in Figure 5. DCT algorithm gives 10.5680 PSNR value, DWT algorithm gives 23.8085 PSNR value, while SLT algorithm gives value of $28.2713 \mathrm{~dB}$ which is slightly same with DWT-DCT algorithm (28.1007 $\mathrm{dB}$ ). The purpose algorithm of this research which is SLT-SCT still gives the best PSNR value (32.6842).

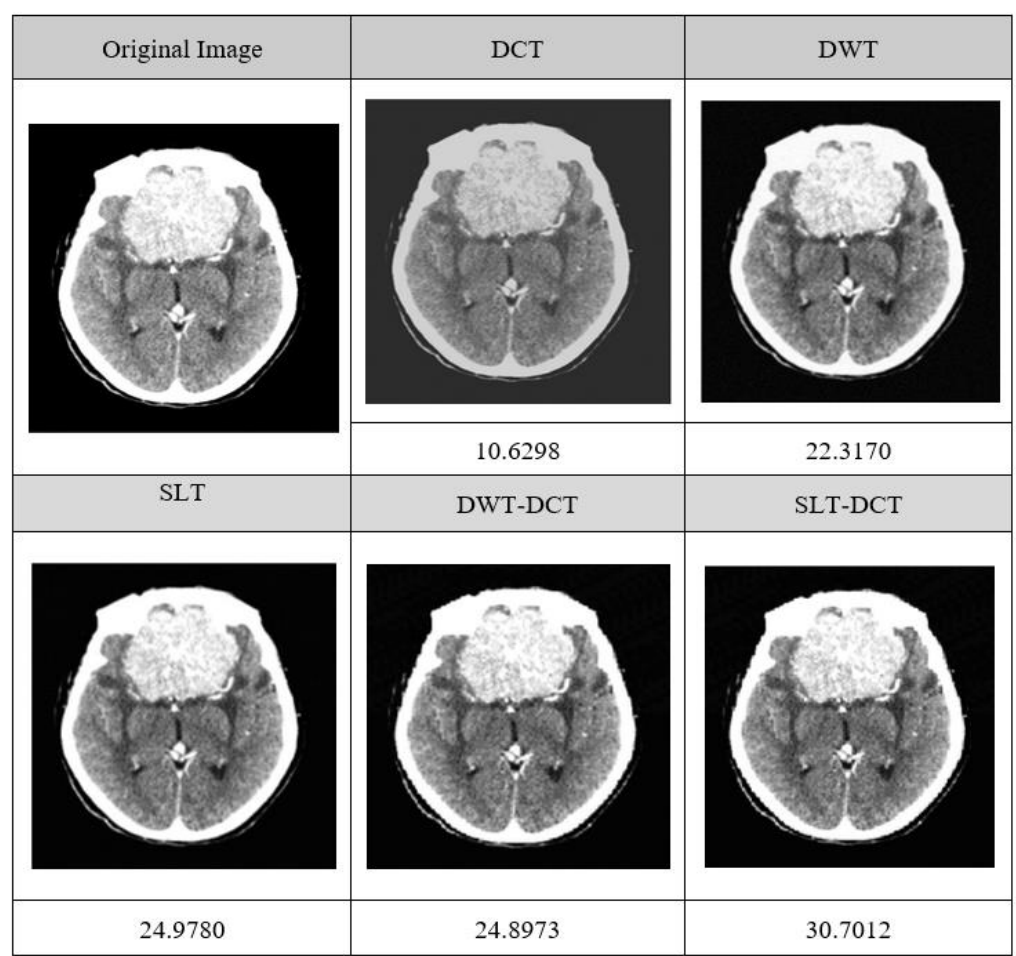

Figure 4. A Result of Stego Images PSNR using Brain_Gray.jpg (in dB)

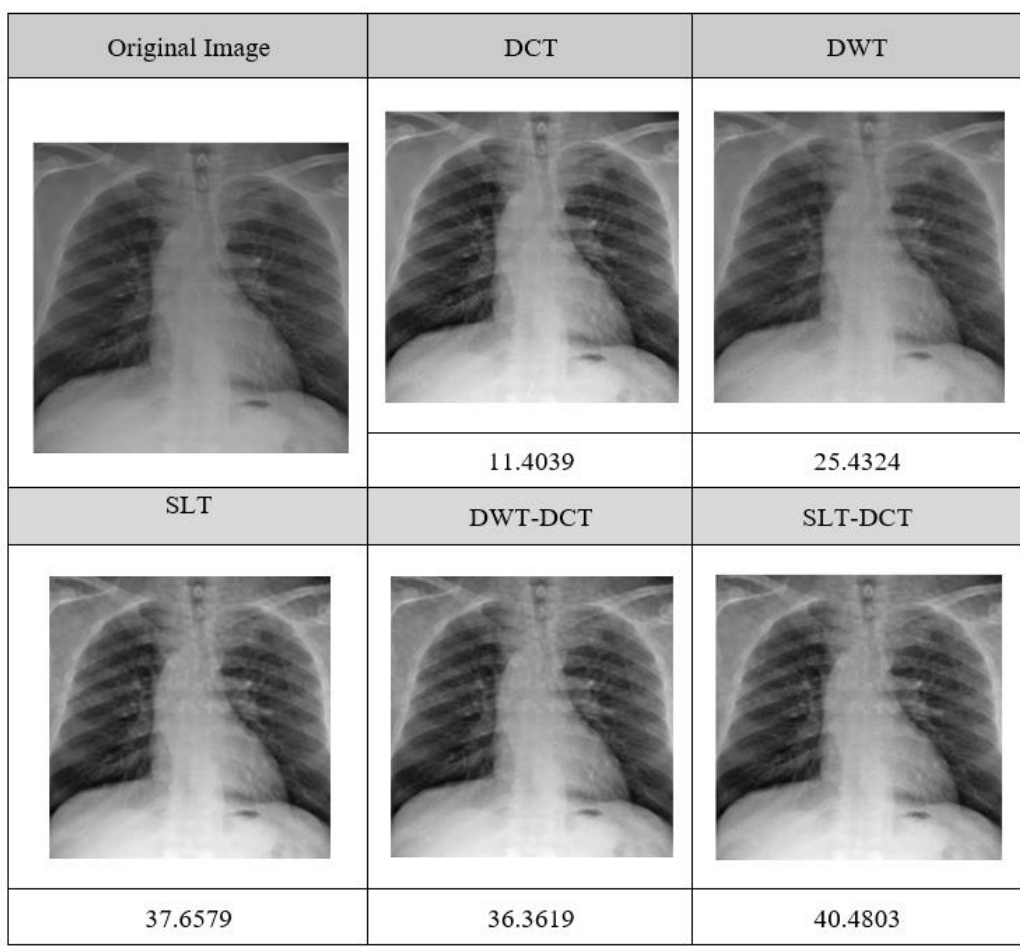

Figure 5. A Result of Stego Images PSNR using Chest_Gray.jpg (in dB)

An Enhancement of Data Hiding Imperceptibility using Slantlet Transform (SLT) Daurat Sinaga, Christy Atika Sari, Eko Hari Rachmawanto, De Rosal Ignatius Moses Setiadi, 


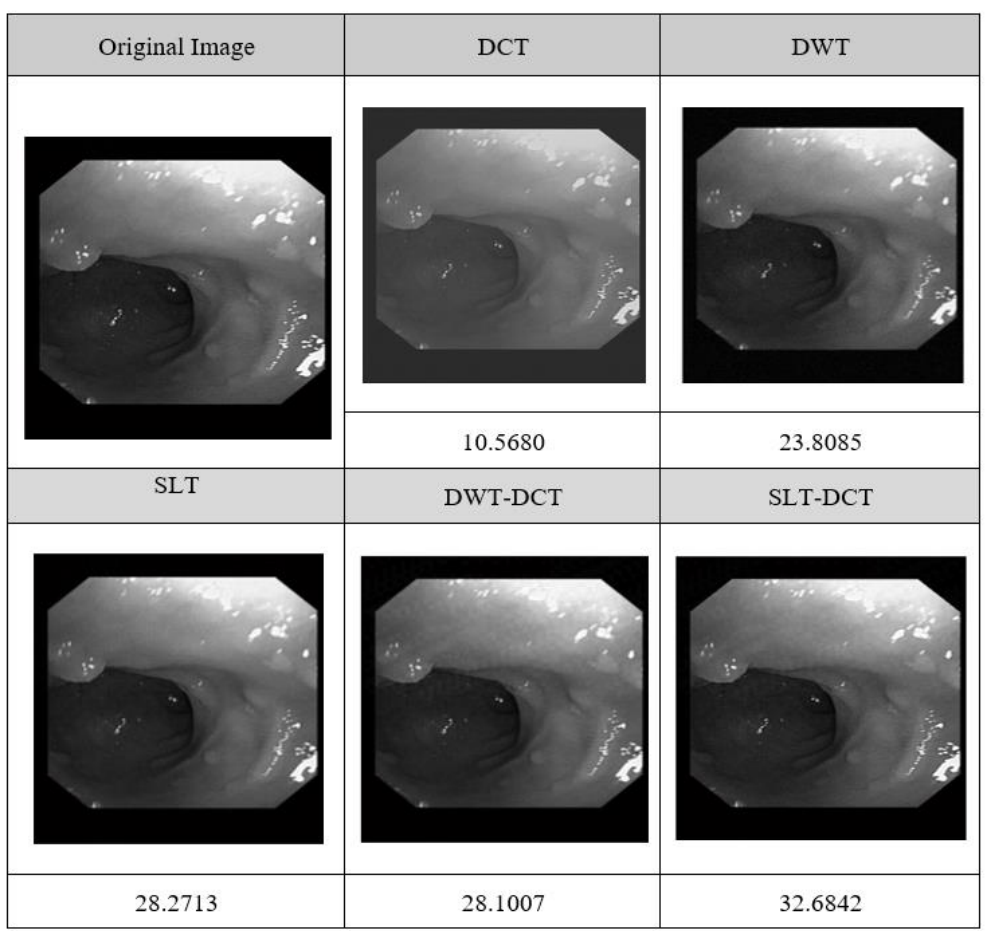

Figure 6. A Result of Stego Images PSNR using Endoscopic_Gray.jpg (in dB)

The result of PSNR value in Endoscopic_Gray.jpg is shown in Figure 6. DCT algorithm gives 10.5680 PSNR value, DWT algorithm gives 23.8085 PSNR value, while SLT algorithm gives value of $28.2713 \mathrm{~dB}$ which is slightly same with DWT-DCT algorithm (28.1007 dB). The purpose algorithm of this research which is SLT-SCT still gives the best PSNR value (32.6842). Figure 7 shows the result of Stego Images PSNR using Larynx_Gray.jpg. Comparing with the other algorithm, the purpose algorithm SLT-DCT still gives the best PSNR value (36.8956 dB). It is proving and strengthening the result that has been explained in Figure 7 until Figure 8.

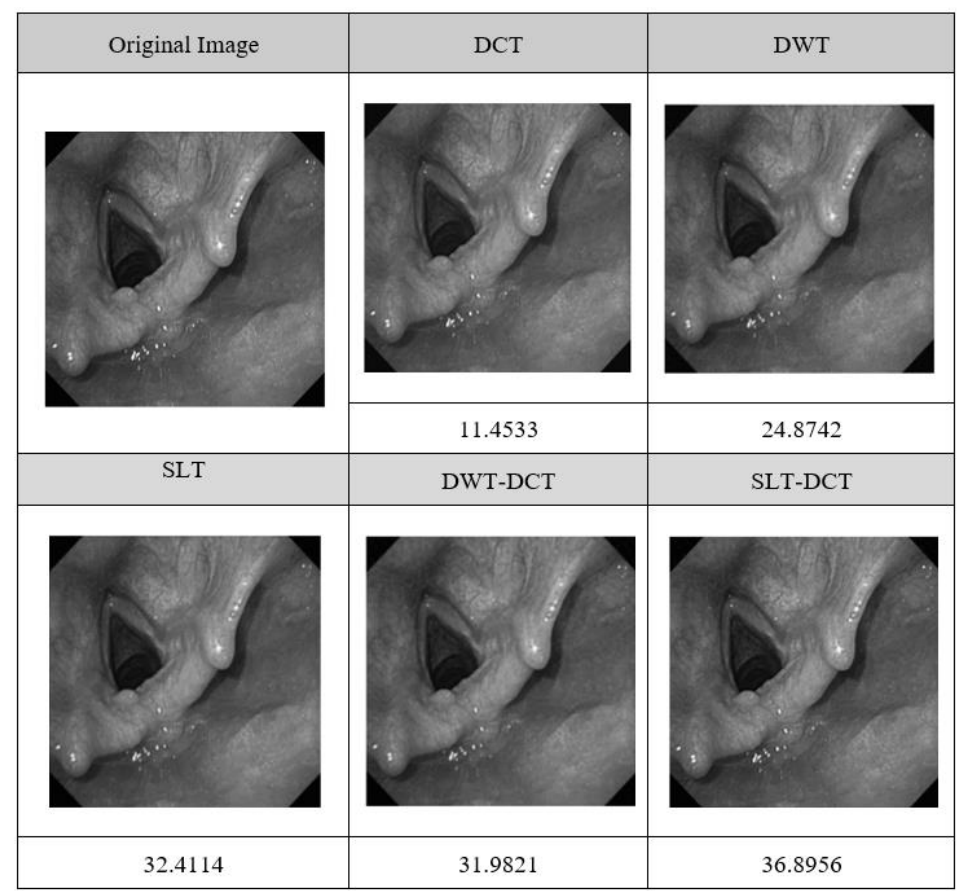

Figure 7. A Result of Stego Images PSNR using Larynx_Gray.jpg (in dB) 


\begin{tabular}{|c|c|c|c|c|c|c|c|c|c|c|c|}
\hline \multirow{2}{*}{$\begin{array}{c}\text { Original } \\
\text { Image }\end{array}$} & \multirow{2}{*}{$\begin{array}{l}\begin{array}{l}\text { Original } \\
\text { message }\end{array} \\
\end{array}$} & \multicolumn{2}{|c|}{ DCT } & \multicolumn{2}{|c|}{ DWT } & \multicolumn{2}{|c|}{ SLT } & \multicolumn{2}{|c|}{ DWT-DCT } & \multicolumn{2}{|c|}{ SLT-DCT } \\
\hline & & Embedd & Extract & Embedd & Extract & Embedd & Extract & Embedd & Extract & Embedd & Extract \\
\hline & tust & \multicolumn{2}{|c|}{. } & \multicolumn{2}{|c|}{ tust } & \multicolumn{2}{|c|}{ hus } & \multicolumn{2}{|c|}{ trișt } & \multicolumn{2}{|c|}{ t) } \\
\hline \multicolumn{2}{|c|}{ Time Taken } & 2.2344 & 1.3438 & 11.7500 & 20.5313 & 9.9375 & 8.3594 & 10.8281 & $\begin{array}{l}9.4688 \\
\end{array}$ & 11.5469 & 9.3750 \\
\hline & tust & \multicolumn{2}{|c|}{ Zust } & \multicolumn{2}{|c|}{ tust } & \multicolumn{2}{|c|}{ tust } & \multicolumn{2}{|c|}{ tust } & \multicolumn{2}{|c|}{ tust } \\
\hline \multicolumn{2}{|c|}{ Time Taken } & 2.3594 & 1.3281 & 11.6875 & 20.7813 & 9.9668 & 8.2656 & 11.2656 & 9.3438 & 11.7188 & 9.7656 \\
\hline & tust & \multicolumn{2}{|c|}{ ișt } & \multicolumn{2}{|c|}{ tust } & \multicolumn{2}{|c|}{ tust } & \multicolumn{2}{|c|}{ tust } & \multicolumn{2}{|c|}{ tust } \\
\hline \multicolumn{2}{|c|}{ Time Taken } & 2.1406 & 1.4219 & 11.7031 & \begin{tabular}{|l|}
21.2188 \\
\end{tabular} & 9.9375 & 8.3594 & 10.7656 & 8.9531 & 10.8438 & 9.0313 \\
\hline & tust & \multicolumn{2}{|c|}{ tust } & \multicolumn{2}{|c|}{ tust } & \multicolumn{2}{|c|}{ tust } & \multicolumn{2}{|c|}{ tust } & \multicolumn{2}{|c|}{ tust } \\
\hline \multicolumn{2}{|c|}{ Time Taken } & 2.0781 & 1.2656 & 11.7813 & \begin{tabular}{|l|l|}
20.6719 \\
\end{tabular} & 10.0781 & 8.4531 & 10.8438 & \begin{tabular}{|l|l|}
9.0313 \\
\end{tabular} & 11.7969 & 9.6875 \\
\hline
\end{tabular}

Figure 8. A Comparison of Extracting Message and Time Taken in Embedding and Extracting Process

Figure 8 describes the comparison of time taken and result of extracting message using five algorithms toward four different images. From the result it can be identified that DWT algorithm gives better extracting message result compare with SLT-DCT. Even SLT-DCT has less quality of extracting message result, the message still can be read easily and the goal of steganography still can be achieved.

From the time taken point of view it can be concluded that the more time needed to do the extracting process, the better extracting message result can be got. SLT, DWT-DCT and SLTDCT take in average 9 seconds to do the extracting process and the result is in medium quality. Comparing with DCT that take in average 1.3 second it gives less quality of the extracting message. DWT give the best quality of extracting message result since it takes the longest time (average 20.5 seconds).

Table 1. A Comparison of PSNR Each Algorithm Using 4 Different Images

\begin{tabular}{cccccc}
\hline Original Image & DCT & DWT & SLT & DWT-DCT & SLT-DCT \\
\hline Brain_Gray.jpg & 10.6298 & 22.3170 & 24.9780 & 24.8973 & 30.7012 \\
Chest_Gray.jpg & 11.4039 & 25.4324 & 37.6579 & 36.3619 & 40.4803 \\
Endoscopic_Gray.jpg & 10.5680 & 23.8085 & 28.2713 & 28.1007 & 32.6842 \\
Larynx_Gray.jpg & 11.4533 & 24.8742 & 32.4114 & 31.9821 & 36.8956 \\
\hline
\end{tabular}

Here, Table 1 indicates the conclusion that SLT-DCT gives the significant result of PSNR value toward four different images compare with the other algorithms. Based on comparison result among the images in SLT-DCT algorithm, the best PSNR value is derived from Chest_Gray.jpg image and the worst PSNR value is resulted from Brain_Gray.jpg image.

Imperceptibility analysis is used to analyze any results obtained from previous experiment, to show the performance of the proposed SLT-DCT steganography algorithm. The PSNR value for Chest in Table 1 reaches 40,4803 dB. Compare with another image that presented in Figure 5,6 , and 7, proved that Chest is highest than another images. In another hand, Brain is the lowest with 30,7012 dB. While Endoscopic PSNR value is 32,6842 dB and Larynx yield 36,8956 dB. Overall of stego image get PSNR value more than $30 \mathrm{~dB}$. PSNR range $\geq 20$ and $\leq 40$ that acceptable to Human Visual System and good quality reconstructed image. Our result can be expressed in Figure 9.

The three stego image also outperform previous work by [9] are Brain, Chest and Larynx. Figure 10 shows the PSNR comparison between proposed algorithm and existing previous works.

Figure 10 describe the comparison of existing algorithm using DWT-DCT algorithm by [11] and new algorithm propose by this research. The existing DWT-DCT algorithm shows better 
PSNR value compare with another algorithm those are DCT and DWT algorithm. The purpose algorithm, SLT-DCT come up to give better result of PSNR value compare with the existing algorithm (DWT-DCT).

For every selected image, the results of stego image are created to ascertain the relation of time taken based on the different type of algorithm. Then, the result of execution time of each data files in the selected image steganography during evaluation process is resumed. The time taken has not only increased speeds but also improved real-time aspects of multicore processing - particularly the predictability of images being be shown in a very short time with little variation in the time taken to process the images. Although the time taken from each image are different, time taken has been the benchmark to analyze of each algorithm.

This experiment result based on time taken show that DCT is lowest time taken (average 1.3 second) than another technique as given in Figure 11. Besides that, DWT show highest time taken (average 20 second). The propose method show that lower time taken than DWT, higher time taken than DCT and also slightly same with SLT and DWT-DCT. Whereas, Figure 12 show a time taken comparison between DCT, DWT, SLT, DWT-DCT and SLT-DCT. This figure state that SLT-DCT yield shorter time than DWT and slightly different with DWT-DCT or SLT only.

Table 2 presents that this proposed method in SLT-DCT better performance than an existing work. Comparing in the same image are Chest and Larynx using same DWT-DCT. In the proposed method, PSNR Chest is $36,3619 \mathrm{~dB}$ that better than PSNR Chest of previous work only $29,58 \mathrm{~dB}$. While PSNR Larynx of existing algorithm is $32,36 \mathrm{~dB}$ that slightly better than proposed method is $31,9821 \mathrm{~dB}$. In another hand, using image Brain and Endoscopic, the PSNR result of DWT-DCT can be categories as good. SLT-DCT as an enhance algorithm achieve great performance better than DWT-DCT both in existing work and this experiment. Especially using image Chest and Larynx which each $40,4803 \mathrm{~dB}$ and 36,8956 dB which is a significant result compare with DWT-DCT in existing work. The others are Brain in 30,7012 dB and Endoscopic in $32,6842 \mathrm{~dB}$. From the time taken point of view it can be concluded that the more time needed to do the extracting process, the better extracting message result can be got. SLT-DCT algorithm require a longer time than DWT-DCT. It is influenced from a hybrid algorithm.

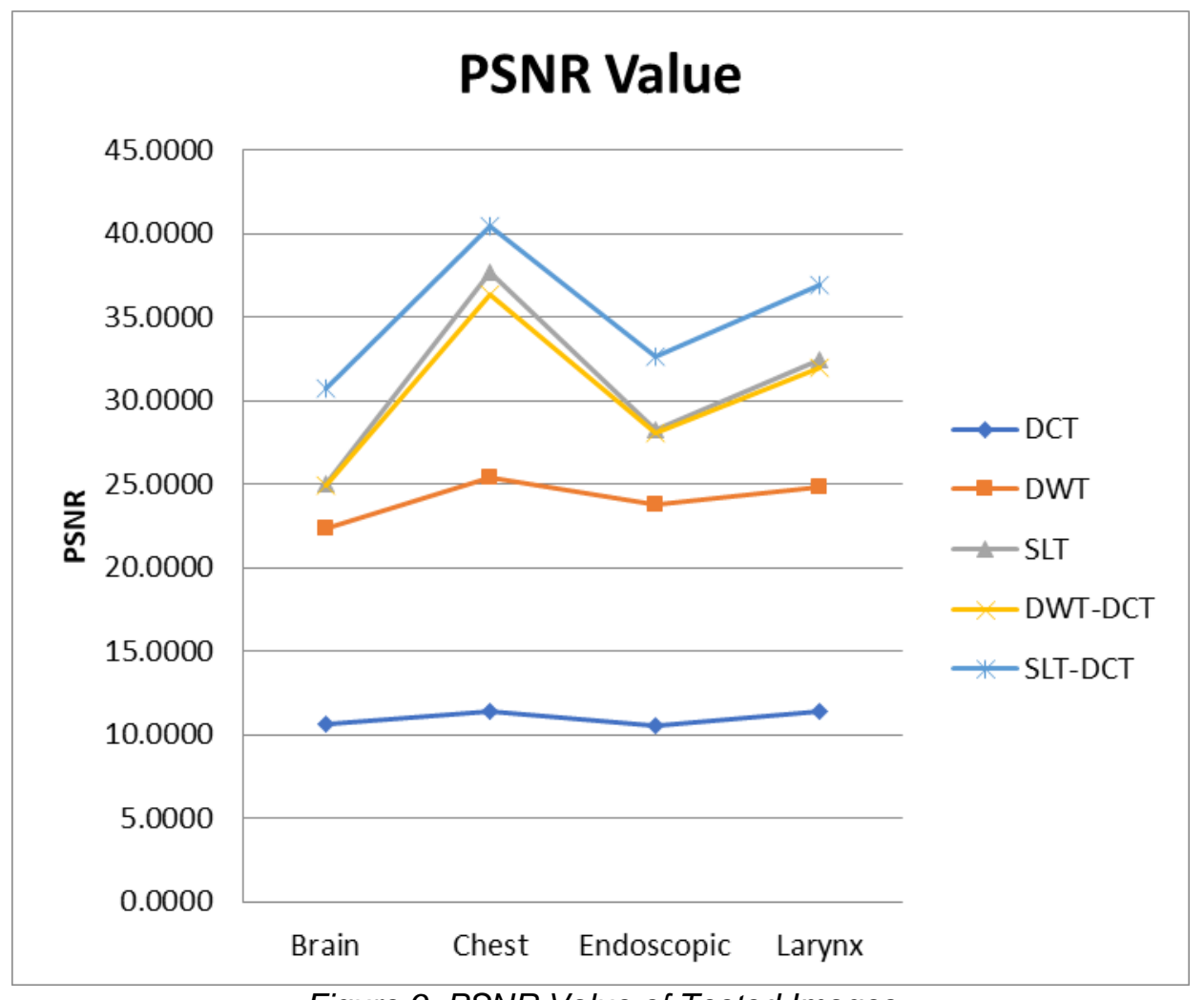

Figure 9. PSNR Value of Tested Images 


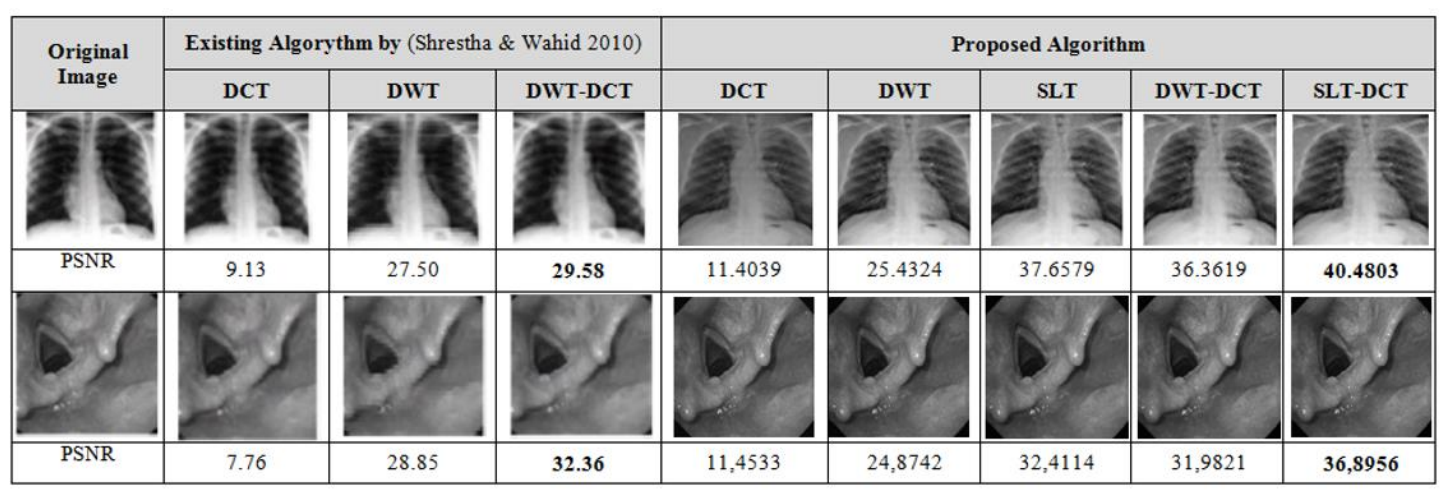

Figure 10. A Comparison PSNR With Existing Algorithm by [10] and the Proposed Algorithm Using SLT-DCT

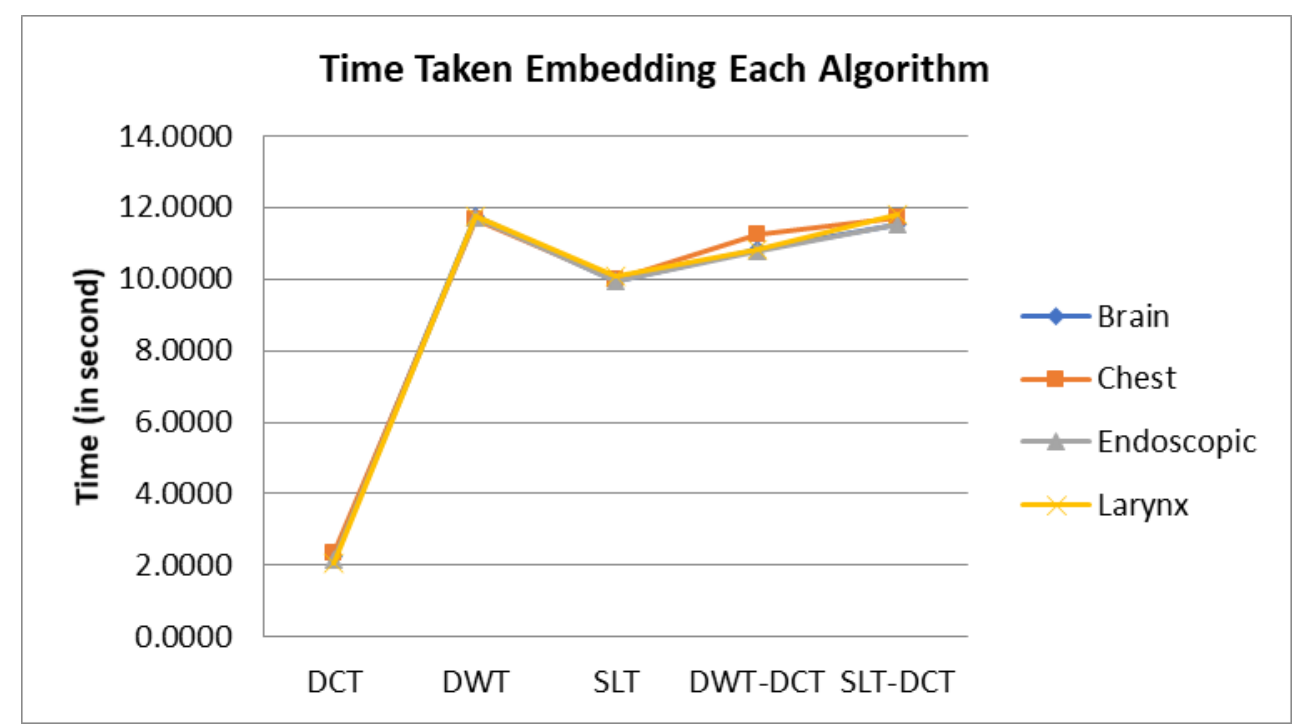

(a) Embedding of Each Algorithm

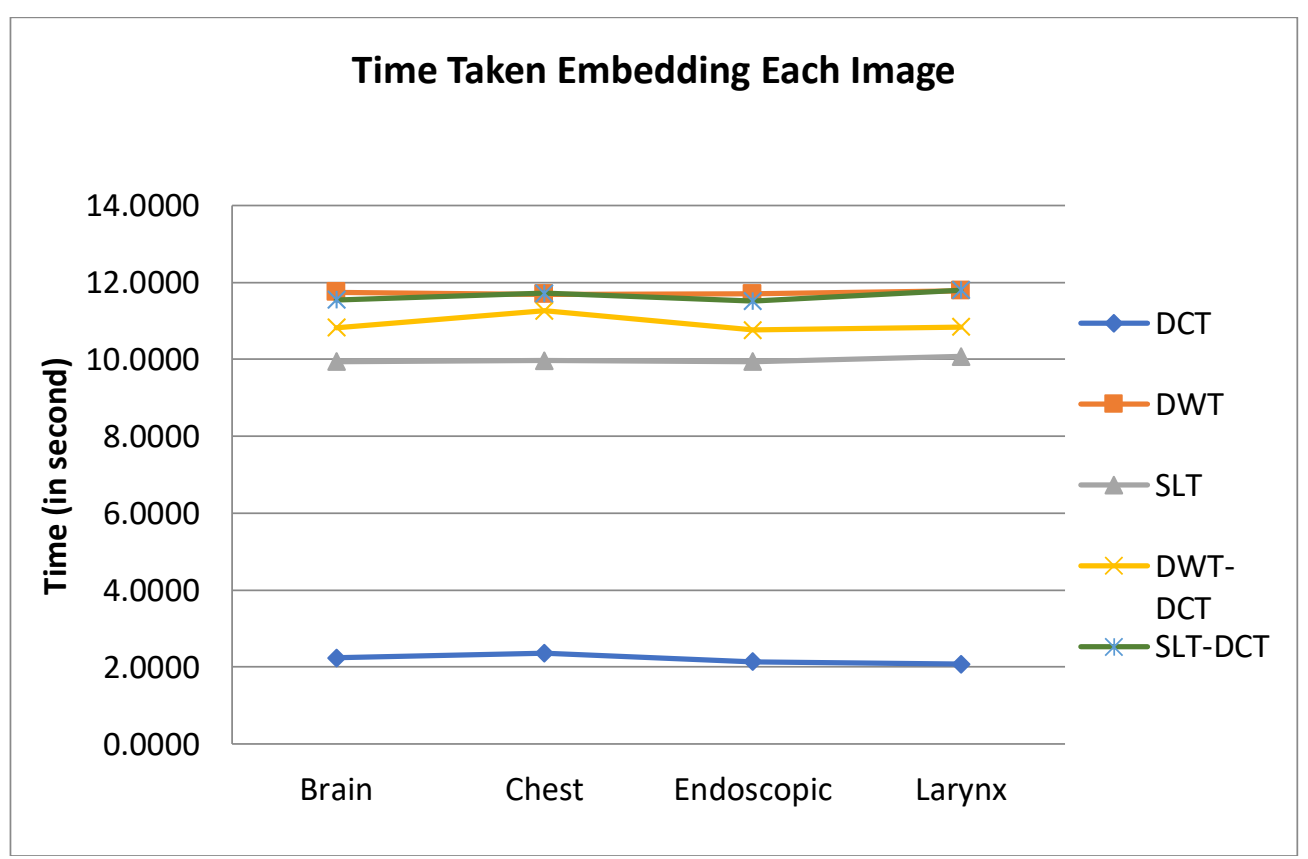

(b) Embedding of Each Image

Figure 11. A Comparison Embedding Time Taken

An Enhancement of Data Hiding Imperceptibility using Slantlet Transform (SLT) Daurat Sinaga, Christy Atika Sari, Eko Hari Rachmawanto, De Rosal Ignatius Moses Setiadi, 
Time Taken Extracting Each Algorithm

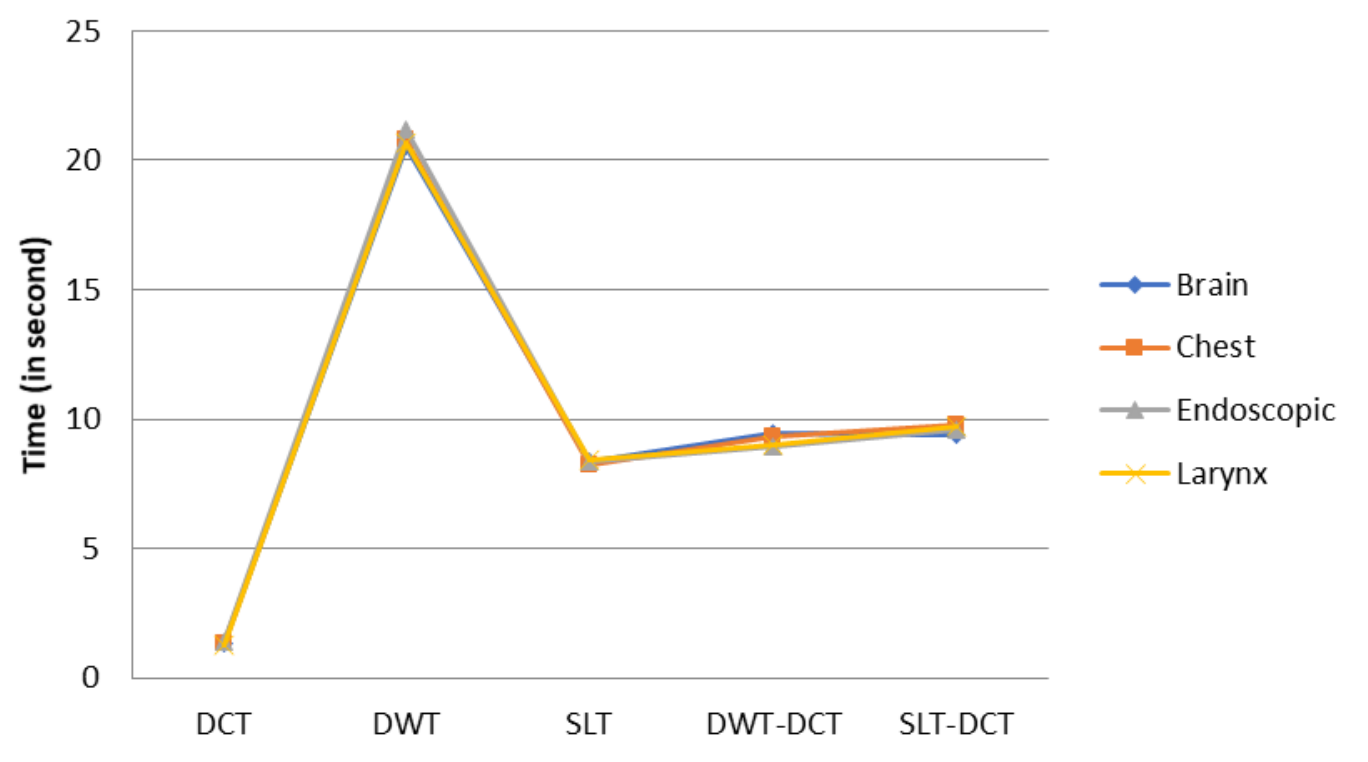

(a) Extracted of Each Algorithm

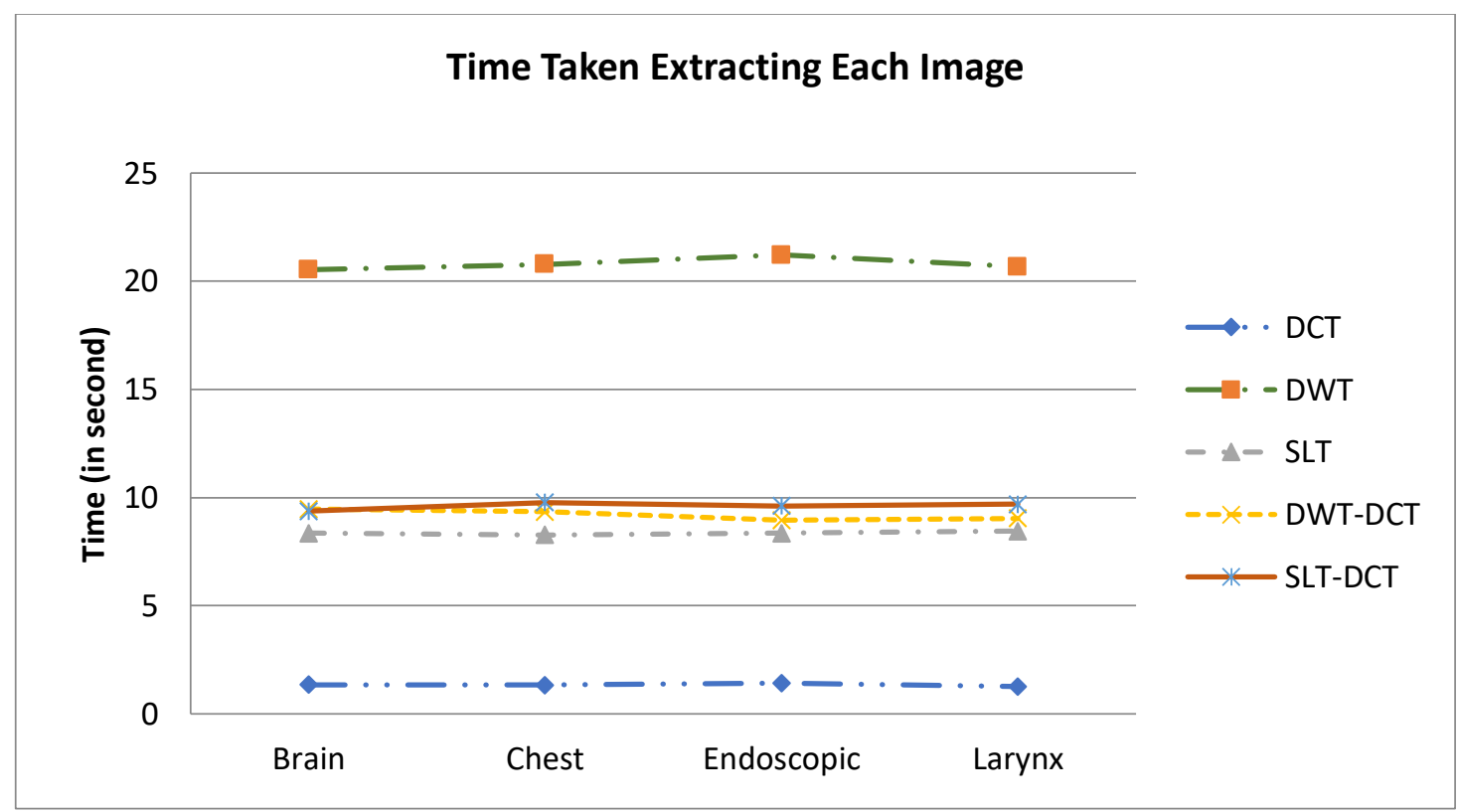

(b) Extracted of Each Image

Figure 12. A Comparison Extracting Time Taken

Table 2. PSNR (in $d B$ ) and Time Taken (in second) Based on DWT-DCT and SLT-DCT Existing Algorithm by

[10]

Proposed Algorithm

\begin{tabular}{cccccc} 
& \multicolumn{1}{c}{ Image } & DWT-DCT & \multicolumn{2}{c}{ DWT-DCT } & \multicolumn{2}{c}{ SLT-DCT } \\
\cline { 2 - 6 } & PSNR & PSNR & $\begin{array}{c}\text { Time Taken } \\
\text { (Extract) }\end{array}$ & PSNR & $\begin{array}{c}\text { Time Taken } \\
\text { (Extract) }\end{array}$ \\
\cline { 2 - 6 } & - & 24,8973 & 9,4688 & 30,7012 & 9,3750 \\
Brain & 29,58 & 36,3619 & 9,3438 & 40,4803 & 9,7656 \\
Chest & - & 28,1007 & 8,9531 & 32,6842 & 9,6094 \\
Endoscopic & 32,36 & 31,9821 & 9,0313 & 36,8956 & 9,6875 \\
Larynx & & & &
\end{tabular}

KINETIK Vol. 4, No. 1, February 2019: 87-98 


\section{Conclusion}

This experiment has been done toward medical image that implemented using medical image. According the research objective in introduction, this experiment was completed and achieved a good performance. The result was conducted to implement on some algorithm such as DCT, DWT, SLT, DWT-DCT and SLT-DCT. In compare to current steganography algorithms which are based on the joint of DWT-DCT, proposed algorithm is achieved significantly higher imperceptibility. Eventually this experiment can be proved that SLT-DCT is the suitable algorithm to securing medical image. While the validation and testing done by PSNR and time taken.

\section{References}

[1] C. Kuo and J. Liu, "Development Od A Web-Based Telemedicine System for Remote ENT Diagnoses," in International Conference on System Science and Engineering, 2010.

[2] A. Shejul and U. Kulkarni, "A DWT Based Approach for Steganography Using Biometric," in International Conference on Data Storage and Data Engineering, 2010.

[3] I. Selesnick, "The Slantlet Transform," IEEE Transactions on Signal Processing, Vol. 47, No. 5, Pp. 1304-1313, 1999.

[4] S. Kumar and S. Muttoo, "Distortionless Data Hiding Based on Slantlet Transform," in International Conference oon Multimedia Information Networking and Security, 2009.

[5] M. Maitra, A. Chatterjee and F. Matsuno, "A Novel Scheme for Feature Extraction and Classification of Magnetic Resonance Brain Image Based on Slantlet Transform and Support Vector Machine," in SICE Annual Conference, 2008.

[6] A. Chatterjee, M. Maitra and S. Goswami, "Classification of Overcurrent and Inrush Current for Power System Reliabikity using Slantlet Transform and Artificial Neural Network, "Expert System Application, Vol. 36, No. 2, Pp. 2391-2399, 2009.

[7] S. Kumar and S. Mutto, "Steganography based on Contourlet Transform," International Journal Computer Science, Vol. 9, No. 6, Pp. 215-220, 2011.

[8] S. Muttoo and S. Kumar, "Secure Image Steganography based on Slantlet Transform," Methods and Models in Computer Science, 2009.

[9] E. H. Rachmawanto, C. A. Sari, Y. Astuti and L. Umaroh, "A Robust Image Watermarking Using Hybrid DCT and SLT," in International Seminar on Application for Technology of Information and Communication, Semarang, 2016.

[10] S. Shresta and K. Wahid, "Hybrid DWT-DCT Algorithm for Biomedical Image and Video Compression Applications," in International Conference on Information Science, Signal Processing and thier Application (ISSPA), 2010.

[11] F. M.A., H. Rahmalan, E. H. Rachmawanto and C. A. Sari, "Impact Analysis for Securing Image Data using Hybrid SLT and DCT," International Journal of Future Computer and Communication, Vol. 1, No. 3, Pp. 308, 2012. 
KINETIK Vol. 4, No. 1, February 2019: 87-98 